\title{
$\underline{\mathbf{P}-132}$
}

\section{Antiproliferative Effect of Antioxidant Rich Fraction Isolated from Cassia Auriculat and its Molecular Mechanisms}

\author{
Samara Kraidi ${ }^{1, *}$, Aishah Adam ${ }^{1}$, Wan Iryani $^{2}$ and Ghanya Al-Naqeb ${ }^{3}$ \\ ${ }^{1}$ Pharamco- Toxicology Research Lab, Faculty of Pharmacy, UiTM, 42300, Bandar Puncak Alam, Selangor; ${ }^{2}$ Cell \\ Signaling Research Lab, Faculty of Pharmacy, UiTM 42300, Bandar Puncak Alam, Selangor; ${ }^{3}$ Department of Food \\ Science and Technology, Faculty of Agriculture, University of Sana'a, Sana'a, Yemen; E-mail: shurshur86@yahoo.com
}

Cassia auriculata is a common south Asian medicinal plant, widely used in traditional medicine for treating various diseases condition and the therapeutic potential has well documented as anti-diabetic, anti-oxidant and anti-hyperlipidemic from different parts of the plant. However, none of the studies have reported the anti-proliferative effect of $C$. auriculata flowers on hepatocellular carcinoma. Thus, ethyl acetate fraction of methanolic extract has been tested for its anti-proliferative activity and ability to induce apoptotic signaling transduction on HepG2 cell line. The cytotoxicity was measured by MTS assay for cellular viability, different stages of apoptotic programed cell death as well as phosphotidylserine externalization were confirmed using annexin- $\mathrm{V}$ and propidium iodide staining using flowcytometry and the molecular mechanisms of cell death were analyzed by RT-PCR. The results showed that EtOAc fraction inhibits the growth of HepG2 cells with an $\mathrm{IC}_{50}$ of $12.5 \mu \mathrm{g} / \mathrm{ml}$, induced cell death in a dose dependent manner and the apoptosis was the main mode of cell death. These effects were attributed to upregulation of $\mathrm{p} 53$, Bax and caspase- 3 by $5.7,7$ and 2.6 fold respectively, and that was associated with suppression of Bcl-2 by $70 \%$. It could be concluded that EtOAc fraction of $C$. auriculata is a promising anticancer agent for hepatocellular carcinoma that could be utilized pharmaceutically.

Keywords: Anti-proliferative, Cassia auriculata, flowcytometry, HepG2, RT-PCR. 